\title{
Acreditación para un programa no convencional por ACCECISO
}

\section{Accreditation for a non-conventional program by ACCECISO}

DOI: $10.46932 /$ sfjdv2n5-141

Received in: Oct 1st, 2021

Accepted in: Dec 30th, 2021

\section{Ma. Raquel Carvajal Silva}

$\mathrm{PhD}$ in Social Scienses

Research Professor at the Universidad de Guadalajara, Centro Universitario de Ciencias Sociales y

Humanidades

Guanajuato 1045, Alcalde Barranquitas C.P. 45100, Guadalajara, Jalisco, México

E-mail:ma.carvajal@academicos.udg.mx

\author{
María Rosas Moreno \\ Master in Social Scienses \\ Research Professor at the Universidad de Guadalajara, Centro Universitario de Ciencias Sociales y \\ Humanidades \\ Guanajuato 1045, Alcalde Barranquitas C.P. 45100, Guadalajara, Jalisco, México \\ E-mail: m.rosas@academicos.udg.mx
}

Anabel Castillón Quintero

$\mathrm{PhD}$ in Social Scienses

Research Professor at the Universidad de Guadalajara, Centro Universitario de Ciencias Sociales y

Humanidades

Guanajuato 1045, Alcalde Barranquitas C.P. 45100, Guadalajara, Jalisco, México

E-mail: anabel.castillon@academicos.udg.mx

\section{RESUMEN}

El presente trabajo da cuenta de la última evaluación realizada al programa de Nivelación al Trabajo Social, por ACCECISO, para obtener una tercera reacreditación, pero donde por primera vez se utiliza una vitrina metodológica hecha para un programa no convencional. En las dos últimas acreditaciones se le evaluó como un programa presencial y las recomendaciones se realizaron a pesar de las diferencias, el programa evolucionó y mejoró de tal manera que el día de hoy espera un dictamen positivo. Las debilidades que encuentre ACCECISO, permitirán que el programa se consolide aún más y que otros de la misma modalidad sean considerados de calidad al igual que cualquier programa convencional.

Palabras clave: evaluación, reacreditación, ACCECISO, programa no convencional, nivelación a una licenciatura.

\section{ABSTRACT}

This paper reports on the last evaluation of the Social Work Leveling program by ACCECISO, in order to obtain a third reaccreditation, but where for the first time a methodological showcase is used for a non-conventional program. In the last two accreditations it was evaluated as a classroom program and the recommendations were made despite the differences, the program evolved and improved in such a way that today it expects a positive opinion. The weaknesses found by ACCECISO will allow the program to be further consolidated and others of the same modality to be considered of quality as any conventional program. 
Keywords: evaluation, reaccreditation, ACCECISO, non-conventional program, leveling to a bachelor's degree.

\section{INTRODUCCIÓN}

¿Qué significa ser un programa acreditado? Que debe funcionar con procesos de calidad y tener disposición para que lo evalúen y mejorar lo ya hace bien. Reconocer lo que falta, lo que se debe modificar y desarrollarse en el tiempo; todo ello definido en un plan de mejora continua que se construya creativa y permanente en el quehacer cotidiano.

Debe estar en la continua autoevaluación para reconocer las oportunidades y los retos diarios que la sociedad demanda. Exponerse a la mirada de otros, de organismo externos que también son validados para realizar estos procesos.

Así, en el contexto de impulsar los sistemas de evaluación en las instituciones de educación superior (IES) encabezados por la SEP y la ANUIES, dieron origen al COPAES, que engloba a diversos organismos acreditadores autónomos en diferentes disciplinas.

El Consejo para la Acreditación de la Educación Superior A.C. (COPAES), por tanto es la única instancia autorizada por el Gobierno Federal a través de la Secretaría de Educación Pública (SEP), para conferir reconocimiento formal y supervisar a organizaciones cuyo fin sea acreditar programas educativos del tipo superior en México, con el objetivo de asegurar calidad educativa, mediante el reconocimiento formal de organizaciones acreditadoras, como es el caso de ACCECISO. Los procesos de acreditación por parte de COPAES, tienen una validez de 5 años e iniciaron en la Universidad de Guadalajara por primera vez en diciembre de 2001.

De entonces a la fecha y de acuerdo a lo establecido en el Plan Institucional de Desarrollo Visión 2030 de la Universidad de Guadalajara, se establece entre sus políticas la pertinencia y la calidad de los programas educativos a través de la producción de información, indicadores y estudios que contribuyan a la cultura de la evaluación y sean puntos de partida para la toma de decisiones de las distintas áreas que sustentan los Centros Universitarios

En este contexto la Nivelación a la Licenciatura en Trabajo Social, en su tercer proceso de reacreditación por ACCECISO, por primera vez se realiza con una metodología específica para la modalidad del programa, en línea y a distancia. 


\section{EL CASO ESPECÍfICO DE LA NIVELACIÓN A LA LICENCIATURA EN TRABAJO} SOCIAL

La población demandante de educación media y superior en Jalisco ha favorecido que la Universidad de Guadalajara consolide la red universitaria para atender el rezago educativo, ampliando su cobertura de educación media y superior.

Consciente de la dinámica social que impera en las concentraciones urbanas, se implementó un programa de nivelación profesional, bajo la modalidad educativa abierta y a distancia para dar respuesta a las necesidades de los técnicos en trabajo social en activo principalmente, de acuerdo con las políticas de nuestra Universidad, que propiciará el desarrollo académico de estos técnicos, sin que la distancia o el tiempo se convirtiera en un impedimento para su mejora.

Tanto en Jalisco como en el resto de los estados de la República Mexicana, debido a los problemas sociales generados por los flujos migratorios, envejecimiento y feminización de la pobreza, polarización del nivel socioeconómico, violencia social y de género, entre otros, se requiere de licenciados en Trabajo Social en las instancias educativas, de salud y de seguridad pública existentes en las instituciones públicas y privadas de todo el país.

Cada una de estas condiciones problemáticas que se han enumerado son ámbitos de análisis e intervención del Trabajador Social, en el entendido de que este profesional cuenta con los saberes metodológicos para el estudio de la realidad social a escalas macro y micro, además de contar con las habilidades para la implementación de políticas y programas sociales, enfocados a la resolución de problemáticas específicas.

Por tanto, el programa de Nivelación cumple con la misión de formar Licenciados en Trabajo Social que tengan un acercamiento teórico-metodológico a las diversas ciencias y disciplinas que comprende el campo de acción profesional, propiciando la reflexión y el análisis de los problemas propios del ejercicio profesional.

La característica del programa de Nivelación es que se imparte en una modalidad a distancia por medios electrónicos, lo que le permite recibir estudiantes residentes de toda la República, incluso del extranjero, y cursen por medios electrónicos o de manera virtual (en línea) sin necesidad de presencialidad.

Esta ventaja operativa del programa lo ha posicionado como el más solicitado en el país, los estudiantes se inscriben en nuestro programa por tres importantes razones: 1) El prestigio, sin lugar a duda, de ser egresado de la Universidad de Guadalajara y 2) Porque siendo adultas y adultos trabajadores, sus posibilidades de tiempo los limita para cursar de forma presencial y, 3) Finalmente, por estar actualmente evaluado en nivel 1 por los CIEES (Comités Interinstitucionales para la Evaluación de la Educación Superior A.C.), así como contar la acreditación (en 2004), la re acreditación (en 2009) y la última evaluación realizada en fechas recientes (febrero de 2017), esperando sus observaciones y dictamen 
final, consideramos será satisfactorio, por haber sido la primera ocasión en que dicho proceso se desarrolló de acuerdo a una vitrina metodológica acorde a la modalidad en línea y a distancia por parte de ACCECISO (Asociación para la Acreditación y Certificación en Ciencias Sociales A.C).

El Programa Académico (PA), de la Nivelación a la Licenciatura en Trabajo Social, es un programa ofertado en la modalidad a distancia-virtual, la población atendida, en su totalidad, son personas adultas insertas en el ámbito laboral, pues es requisito de ingreso ejercer la profesión de trabajo social, ya que son personas que cuentan con carrera técnica. Al ser a distancia y virtual, los estudiantes, en su mayoría, radican en varios estados de la república. Sin embargo, el diseño curricular de la Nivelación permite ofrecer el acompañamiento a los estudiantes en la profesionalización de su actividad laboral a través de las actividades planteadas. Así se desarrollan proyectos, prácticas, consultorías y asesorías, en las instituciones donde laboran que están insertas en los ámbitos tanto público como privado.

De acuerdo a una investigación realizada a los egresados del PA en el año $2013^{1}$, las competencias extracurriculares que los estudiantes reconocen haber desarrollado al inscribirse a la Nivelación en esta modalidad, se destacan aquellas que se integran a los rubros: deber hacer, querer hacer, poder hacer, saber hacer, hacer-ser. Estas competencias desarrolladas como parte del currículum oculto dan cuenta de aquellas que contribuyen al emprendurismo de los estudiantes a través de lo aprendido al cursar la Nivelación.

El programa de nivelación a la Licenciatura en trabajo social se difunde a través del portal de la Universidad de Guadalajara por medio de la página web del Centro Universitario de Ciencias Sociales y Humanidades. Existen varios mecanismos para difundir el programa: a través de página web (http://www.cucsh.udg.mx), en toda la república mexicana por medio de tríptico ${ }^{2}$ y cartel el cual es distribuido también en la red universitaria. El tríptico es enviado a los interesados a través de correo electrónico. La difusión también se lleva a cabo de boca a boca por los propios egresados.

El principal medio de convocatoria para este programa es el correo electrónico (propedeuticonilits@yahoo.com.mx), por el cual se especifican tiempos para inscripciones, requisitos y costos para poder ingresar. Los registros se realizan dos veces al año con base a los calendarios escolares.

En el dictamen de Nivelación, están descritos los requisitos para que los estudiantes ingresen al programa de licenciatura ${ }^{3}$ Además en el Artículo 4 del reglamento de la Nivelación a la Licenciatura en trabajo Social ${ }^{4}$ se describen los requisitos indispensables para aspirar a la Universidad de Guadalajara:

- Solicitar el ingreso de acuerdo con la guía de aspirantes que al efecto expida la Universidad;

\footnotetext{
${ }^{1}$ Barragán De Anda, Berenice; De Aguinaga Vázquez, Patricia; Ávila González Claudia. La Educación virtual y los aprendizajes del currículum oculto. Ponencia presentada en el Congreso Ancademia Journals en noviembre de 2013.

2 Tríptico, en físico

${ }^{3}$ Dictamen de la nivelación. Evidencia entregada por la coordinación de carrera

${ }^{4}$ Reglamento del programa NILITS. Evidencia entregada por la coordinación de carrera
} 
- Ser aceptado mediante proceso de admisión;

- Acreditar capacidad suficiente para los estudios que aspira;

- Cubrir la aportación económica correspondiente, y

- Las demás establecidas por el dictamen que aprueban el correspondiente Plan de Estudios.

Como requisito documental, ingresa quien tenga el título de técnico en Trabajo Social, que se encuentre en ejercicio profesional, y que cuente con certificado de bachillerato, así como que demuestre un manejo básico de la Internet.

Además de esta condición básica, existen otros criterios que el programa establece durante el proceso de selección para que el aspirante sea aceptado o rechazado como estudiante.

El dictamen del programa autoriza ofrecer un curso propedéutico que sirve para seleccionar al aspirante, así como una inducción a la forma de trabajo de la modalidad, que debe evidenciar capacidad académica para cursar la Nivelación y se demuestra con esto que se lleva con Equidad esta selección.

El curso propedéutico se estructura en dos partes:

Proceso de inducción a plataforma MOODLE. En este espacio, se les introduce a los aspirantes para que conozcan el manejo de la plataforma Moodle, ya que será una herramienta de interacción entre la comunidad de aprendizaje (Asesor- estudiante. Estudiante - estudiante, estudiantes - asesor). También conocen acerca de la Universidad de Guadalajara, su historia, su misión y visión. Al modelo educativo siglo XXI, del cual se extrae el fundamento teórico del proyecto curricular del programa de nivelación.

2.- Inducción a la modalidad educativa a distancia. Durante el curso propedéutico ${ }^{5}$ se ofrecen los elementos metodológicos de la educación a distancia, conocimientos básicos del manejo de las tecnologías para la comunicación, estrategias de aprendizaje, normas para escribir correctamente, reglamento del programa, para que el aspirante pueda cursar exitosamente una vez que es admitido.

Durante el propedéutico se aplica también una entrevista que aporta datos para la toma de decisiones en el momento de determinar quiénes aprueban o no el proceso ${ }^{6}$.

Este curso se lleva a cabo durante 22 horas presenciales en la ciudad de Guadalajara (o el curso se puede ofrecer en la ciudad de origen de las aspirantes que se constituyen en "grupos"). Después se continua en un proceso de cinco semanas a distancia mediados por la plataforma Moodle (Cursos en línea) o por los materiales enviados por correo electrónico (Modalidad electrónica). Durante ese tiempo el aspirante debe mantener contacto con su asesor para el desarrollo de un trabajo que permitirá evidenciar las capacidades académicas y la apropiación de las formas de trabajo en un sistema no presencial.

\footnotetext{
${ }^{5}$ Curso propedéutico del programa de nivelación en línea: http://www.cenlinea.cucsh.udg.mx/course/view.php?id=1386

${ }^{6}$ Formato de entrevista
} 
Al finalizar el tiempo previsto para el desarrollo del trabajo propedéutico, se reúne una comisión dictaminadora que emite su opinión tomando en cuenta los elementos aportados por la entrevista, las observaciones del asesor del módulo y el trabajo del propio aspirante. Se recoge el resultado de la discusión en una ficha de indicadores para la dictaminación que sistematiza los elementos indispensables para que un aspirante sea admitido. La discusión en casos controversiales es siempre colegiada evitando con esto la decisión unipersonal o el trato desigual o discriminatorio para ninguna persona.

Si los aspirantes acreditan el curso propedéutico se expide una carta de aceptación desde la jefatura del departamento y la coordinación de carrera, misma que se integra al expediente que el aspirante entrega en la coordinación de Control escolar del CUCSH dentro de los plazos para ello establecidos. Solo se reciben expedientes completos.

Los dictámenes de admisión de todos los programas de la Universidad se publican por medios electrónicos, en la página de la Coordinación de Control Escolar de la Universidad en www.escolar.udg.mx y en todas las ligas relacionadas con Centros Universitarios. Además, la difusión física se lleva a cabo en la Gaceta Universitaria que se publica los lunes. Los estudiantes a distancia prefieren consultar el dictamen mediante la página electrónica, dada su remota ubicación geográfica

El programa educativo determina como perfil de ingreso: Interés y responsabilidad en el trabajo, metas definidas, capacidad en el estudio independiente, autodisciplina, capacidad de autocrítica y deseos de superación.

Para cursar el programa de nivelación se requiere: ser trabajador social técnico en ejercicio profesional y que hayan cursado el bachillerato, tener título de trabajador social técnico, cumplir con los requisitos establecidos en el departamento escolar de la Universidad de Guadalajara, así como cursar un propedéutico con el cual se valora el perfil del estudiante.

El plan de estudios de la nivelación está constituido por 12 módulos, cada módulo contiene un conjunto de lecturas como base teórica y un diseño instruccional (guía didáctica) a través de la cual se solicita a los estudiantes vincular la teoría revisada en cada curso con su práctica profesional, por ello es requisito indispensable que las y los estudiantes se encuentren en ejercicio profesional para que puedan analizar y sistematizar su práctica de manera fundamentada. Entre los objetivos del plan de estudios se señala:

Facilitar aquellos elementos que generen la reflexión y análisis del quehacer profesional, a través de un proceso intencionado que replantee sus desempeños en esta disciplina, propiciando:

- La criticidad de los métodos de trabajo empleados

- La aprehensión de referentes teóricos-epistemológicos que expliquen los problemas a los que se enfrenta cotidianamente.

- La búsqueda de nuevos modelos de intervención. 
En cuanto al perfil de egreso al igual que el perfil de ingreso y plan de estudios, se les da a conocer a las estudiantes en el propedéutico de manera general, exponiendo las competencias que adquirirán en la nivelación, así como los conocimientos generales, las habilidades, actitudes, aptitudes y valores. Durante el transcurso de la nivelación se cuida la congruencia entre todos estos elementos explicados claramente en el proyecto curricular de la nivelación. ${ }^{7}$

En anteriores promociones, los aspirantes al programa de nivelación se registraban en una lista de espera para ingresar al curso propedéutico; a los inscritos en los primeros 40 lugares se les proporcionaban un lugar para integrarse al propedéutico sin previa inducción. Pero en los últimos cinco años hubo un cambio que impacta directamente en la política de la Universidad de Guadalajara: ampliar la matrícula sin que se afecte la calidad, por lo que se decidió que del total de aspirantes inscritos en lista de espera, se hace una valoración inicial al 100\% de ellos, a través de un curso introductorio ubicado en la plataforma Moodle, donde se aprecia el manejo de la plataforma, comprensión de contenidos (Conocimiento de la Universidad, Acercamiento disciplinar y habilidades para el análisis). ${ }^{8}$

Las actividades de este curso de inducción se planifican, a partir de la conformación de una base de datos con los interesados durante alrededor de tres meses, y se llevan a cabo designando a cada aspirante un asesor que da seguimiento a sus construcciones para que llegue al curso presencial en igualdad condiciones y conocimientos que el resto del grupo, además de evidenciar en los talleres establecidos, su aprendizaje y apreciación en cuanto a las habilidades que cada aspirante posee para desarrollarlas durante el presencial y posteriormente en el curso propedéutico de cinco semanas.

Para cursar el propedéutico, la selección se realiza con base a los aspirantes que hayan resuelto las actividades solicitadas en la inducción a la plataforma Moodle y que hayan demostrado habilidades para la modalidad a distancia: y quienes tuvieron buen desempeño se les proporciona un lugar en el curso propedéutico para ingresar al programa NILITS. Este mismo procedimiento se lleva a cabo con grupos foráneos (los asesores salen a diferentes estados de la república a impartir el curso propedéutico para el proceso de selección). ${ }^{9}$

Toda la información referente a la inducción sobre el programa y el modelo educativo se les proporciona a los estudiantes durante el curso propedéutico, antes de ser dictaminados. En el curso taller presencial se les imparten los elementos teóricos sobre las generalidades del programa educativo es decir, enfoque teórico y método de enseñanza, en un taller denominado Generalidades del programa de Nivelación a la Licenciatura en Trabajo Social.

\footnotetext{
${ }^{7}$ Proyecto curricular de la nivelación.

8 Proceso de inducción a plataforma moodle: http://www.cenlinea.cucsh.udg.mx/course/view.php?id=1254

${ }^{9}$ Ejemplo de acta de valoración del curso propedéutico
} 
El modelo educativo se les presenta en la primera parte del curso propedéutico en el espacio denominado conocimiento de la Universidad. La URL para consultar el modelo educativo es: http://www.cenlinea.cucsh.udg.mx/mod/page/view.php?id=57179

Lo referente a la normatividad se les da a conocer a los estudiantes durante el curso propedéutico; se programa un taller para explicar el Reglamento de Nivelación en Trabajo Social ${ }^{10}$. De tal forma que cuando ingresan a la nivelación ya tienen el conocimiento sobre la normatividad del programa.

Con relación a mecanismos de participación estudiantil por ser alumnos foráneos distribuidos en toda la república no participan en órganos colegiados y de gobierno, no obstante, se está consideran implementar acciones que permitan a los estudiantes participar como consejeros universitarios y que el programa de nivelación tenga representatividad en dichos órganos de gobierno.

En el programa de Nivelación no se realiza análisis de trayectorias por escuela de origen debido a que los estudiantes del programa son técnicos en trabajo social procedentes de todo el país y en su mayoría tienen de 10 a 15 años de egresados. Algunas de esas de Trabajo Social ya desaparecieron. Sin embargo, si se implementan acciones preventivas y remediales para evitar deserción y reprobación, ejemplo de ello es el programa de tutoría académica.

Los aspirantes se registran dos veces al año para ingresar a la Universidad de Guadalajara, y es ésta quien dictamina estudiantes en dos calendarios A y B. En cada ciclo escolar (A y B), el programa acepta a 45 aspirantes en propedéutico local (Ciudad de Guadalajara) y de 25 a 30 aspirantes foráneos (Sede en diferentes estados de la república mexicana). En cada ciclo escolar se acepta un aproximado de 70 estudiantes.

Para poder ingresar al programa educativo, los aspirantes interesados se registran al correo electrónico propedéuticonilits@yahoo.com.mx y quedan registrados en una lista de espera. De esta lista, la coordinación de carrera responde e instruye a los aspirantes quienes cursarán el propedéutico, ya descrito anteriormente. Como ya se dijo también se valoran las habilidades y competencias para cursar en la modalidad a distancia, y se selección a aquellos que mejor se desempeñaron en el curso y que cubren el perfil de ingreso. Los aspirantes aprobados continúan con sus trámites administrativos en la unidad de control escolar.

En los últimos cinco años la relación de aspirantes y dictaminados ha sido más o menos constante, ejemplo de ello tenemos que en el año 2010 ingresaron 128 estudiantes de un listado de 136 aspirantes; en el 2011 se aceptaron 109 de un listado de 127; en el 2012, ingresan 101 de 125 y en 2013 ingresan 90 de 159. Para el 2014, de 137 aspirantes ingresaron 127, y en 2015 de 108 aspirantes ingresaron 93. Como se puede observar existe una constante con relación al número de aspirantes y los ingresos por dictamen,

\footnotetext{
${ }^{10}$ Reglamento de la Nivelación en Trabajo Social
} 
a diferencia del 2013, que es notable la diferencia entre los inscritos y los admitidos. Cabe mencionar que no todos los aspirantes en lista ingresan al propedéutico porque algunos de ellos desisten antes del curso ${ }^{11}$. La matrícula actual de la nivelación es de: 560 estudiantes

I. TRAYECTORIA ESCOLAR. EL PROGRAMA CUENTA CON UN REGISTRO GENERAL DE LA TRAYECTORIA ACADÉMICA DE CADA ESTUDIANTE. EN DICHO REGISTRO SE TIENE LA INFORMACIÓN DESDE EL PRIMER DICTAMEN EMITIDO EN DICIEMBRE DE 1994 HASTA EL ACTUAL, EN FEBRERO DE 2017 SE EMITIERON UN TOTAL DE 44 DICTÁMENES.

Sobre el sistema de información y trayectoria utilizado es una base de datos en Excel fidedigna y actualizada a la fecha con información precisa e inmediata para verificar: nombre del estudiante, código, número de dictamen al que pertenece, porcentaje de avance por módulo, estatus actual del alumno, (Activo, baja y titulado) calificaciones por módulo y servicio social. Por lo tanto, se tiene control sobre la trayectoria escolar de cada uno de los estudiantes de este programa académico.

En el programa de Nivelación, aunque es abierto y a distancia, desde 2003 se estableció tiempo límite máximo para cursar los módulos de la licenciatura.

En virtud de lo anterior, el estudiante determina el tiempo que invierte en su trayecto por el programa pudiendo ser desde dos años hasta un máximo de 6 años. El promedio actual es de 2 años cursando en línea, con vistas a establecer una estrategia de disminución del tiempo.

Cuando el estudiante decide llevar el curso en línea, la duración por módulo es de cuatro semanas; cuando decide cursarlo en electrónico, la duración máxima para concluir es de uno por ciclo escolar (un semestre).

Considerando lo anterior, es posible afirmar que en los últimos cinco años no ha habido deserción. Y por las características del programa no se puede presentar un índice de rezago como tal, debido a que el programa es flexible para el tránsito de sus estudiantes, sin embargo se manejan tiempos deseables como mínimo de dos años y como máximo de seis para el egreso del alumno que como ya se mencionó dependerá de la modalidad que el estudiante elija (electrónica o en línea).

Se reconoce que por la multiplicidad de actividades que desarrolla el estudiante en sus diversos roles como adultos con responsabilidades familiares, laborales y escolares, el avance académico de cada estudiante tiene un ritmo diferente, pero esto es propio de la modalidad educativa abierta y a distancia.

En el programa, el índice de reprobación es nulo debido a que cuando el estudiante no acredita un módulo, cuenta con la oportunidad de presentarlo a sus asesores antes de mandarlo a calificar hasta recibir la asesoría necesaria para presentar un trabajo que pueda ser acreditado, según consta en el Reglamento vigente del Programa.

\footnotetext{
${ }^{11}$ Dictámenes y cuadro de porcentaje de aspirantes por ciclo escolar.
} 
Programa de tutoría. El programa educativo cuenta con un programa de tutoría con el propósito de acompañar al estudiante durante su proceso académico. El tutor es la figura que acompaña al estudiante para que no se detenga en su trayectoria académica, además de motivarlo está al tanto de situaciones personales que puedan dificultar el avance en los estudios. El 100\% de los estudiantes cuentan con un tutor desde el momento de ingresar al programa. La función del tutor es orientar desde el ingreso y durante toda la trayectoria en circunstancias administrativas, académicas y personales. ${ }^{12}$

Actualización de programas y contenidos de los cursos (módulos). El plan de estudios es modular, en total son 12 módulos los que cursa el estudiante para lograr el grado de licenciatura. Cada módulo cuenta con un grupo de profesores (de 3 a 4) que se reúnen en academias para actualizar el contenido del curso que imparten, se trabaja de manera colegiada y constantemente se revisa guía y antología, así como recursos de apoyo (estrategias didácticas, referencias bibliográficas) de tal forma que los contenidos son actualizados de forma permanente. ${ }^{13}$

En el programa educativo no se conforman grupos como se hace en un sistema presencial.

El programa educativo de la nivelación ofrece dos modalidades para cursar los módulos.

I.- Modalidad electrónica, para ello la coordinación de carrera asigna a cada profesor un listado de estudiantes con los que trabajará por medio de esta modalidad y el medio de interacción es el correo electrónico.

II.- Modalidad en línea a través de plataforma Moodle, donde se le asigna a cada profesor un listado de estudiantes que varía entre 10 y 15 alumnos, donde se considera al grupo como una comunidad de aprendizaje. La interacción se lleva a cabo a través de foros de debate y foros de exhibición, una de las herramientas principales es la guía didáctica.

La cantidad de estudiantes por profesor varía en cada ciclo y en cada módulo, siendo un promedio entre 10 y 20 estudiantes por profesor.

Titulación. Existen diferentes modalidades de titulación reglamentadas en el Reglamento General de Titulación y del Plan de Estudios de la Universidad de Guadalajara, sí como del Reglamento de la Nivelación a la Licenciatura en Trabajo Social. Cabe mencionar que hasta el ciclo escolar 2014 B existían sólo dos modalidades de titulación: excelencia académica y tesis, pero que esto permitía cierto rezago y con la intención de evitarlo y lograr mayor eficiencia terminal, se implementaron más modalidades de titulación. Así a partir del ciclo escolar 2015 A se aprobaron por el H. Consejo de Centro las modalidades de: Examen General de Certificación Profesional (CENEVAL), Trabajo Monográfico de actuación, Seminario de Investigación e Informe de prácticas profesionales

\footnotetext{
12 Programa de tutoría de NILITS, evidencia en la coordinación de carrera

${ }^{13}$ Cursos en plataforma y actas de academia, evidencia en la coordinación de docencia
} 
El objeto de ampliar las modalidades fue que el programa fuera más eficiente al permitir que los alumnos concluyan de la mejor manera su licenciatura fortaleciendo sus habilidades y conocimientos que aplicará en el campo de acción sin dejar de lado valores y actitudes de responsabilidad, ética, criticidad, reflexión, disciplina y compañerismo. Y uno de los parámetros fundamentales que evidencia la eficiencia del Programa es, sin duda, la titulación de los estudiantes.

Con referencia a la eficiencia terminal, los índices de titulación por cohorte generacional, no se pueden calcular como los presenciales en suficiente tener el ingreso de la generación, el tiempo curricular para titularse y el número de titulados, sí, pero en este programa no se calcula de esa manera.

En cada ciclo escolar ingresan al programa alumnos que, por dictamen, tienen un plazo máximo de un ciclo para cursar cada módulo. Sin embargo, al tratarse de un programa abierto y a distancia, se puede cursar cada módulo tan rápido como quieran o realizar un solo modulo cada 6 meses, razón por la que no se puede establecer un tiempo estándar para cursar toda la nivelación, sino que cada estudiante define su ritmo particular.

Por lo anterior, los estudiantes que ingresan en un mismo dictamen, no se titulan al mismo tiempo ni en plazos predeterminados.

Con base en lo anterior se han calculado los índices de titulación en relación al número de ingresos en el ciclo escolar de dos años atrás y los titulados en el presente ciclo, aun sabiendo que no todos los titulados corresponden a los mismos alumnos.

Bajo este procedimiento, los índices de titulación durante los últimos cinco años, contemplando los ciclos escolares 2010 B a 2015 A el promedio es del 45.15\%. Desde luego que fluctúa como se puede observar en 2010 A que fue del 88.67\%, excelente. Durante los últimos calendarios se obstaculizó el proceso de titulación a causa de un retraso en la autorización de los libros de actas.

Las modalidades de titulación más recurrentes en los últimos cinco años han sido titulación por tesis en un $20 \%$ y excelencia académica con un $80 \%$.

Con relación al egreso, calculada por ciclo escolar y no por generación, en los últimos 5 el egreso (quien ingresa y egresa de un mismo dictamen) es del 38\%. Es importante mencionar que los estudiantes cuando concluyen con el plan de estudios terminan el programa porque a la vez que cursan los módulos "X” Métodos de Investigación Social y "XI” correspondiente al de Integración están construyendo sus tesis.

Si vemos el egreso por cohorte generacional de los últimos cinco años se puede observar cómo aumenta la titulación $45.15 \%$, puesto que en cada ciclo escolar egresan y se titulan estudiantes que ingresaron en diferentes ciclos como se explicó anteriormente.

Dado que la evaluación es una actividad que exige compromiso, dedicación, sistematicidad y empeño, además de ser una disposición del Reglamento General de Evaluación y Promoción de Alumnos 
de la Universidad de Guadalajara ${ }^{14}$ y en particular el Reglamento de la Nivelación de la Licenciatura en Trabajo Social, ${ }^{15}$ Sistema Abierto y a Distancia del Programa de Nivelación a la Licenciatura en Trabajo Social, los docentes tienen una función importante en las tareas de evaluación y ésta se toma con mucha seriedad evitando hacer de este proceso una práctica improvisada.

El Programa, según lo señalado en el proyecto curricular, ofrece al alumno un programa virtual y a distancia diseñado para operar con el sistema de créditos y por competencias, favorece métodos de enseñanza que permiten al estudiante adquirir competencias laborales que aumentan su capacidad de respuesta dentro de la vida productiva institucional, local y nacional.

Por otra parte, el sistema de evaluación implementado en el Programa es coherente con el proceso de enseñanza - aprendizaje, teniendo presente que el modelo está orientado al aprendizaje y centrado en el estudiante.

El plan de estudios del programa de Nivelación está basado en una organización modular. El abordaje de los módulos se constituye por unidades de aprendizaje denominadas objetos de estudio. Con base en lo anterior, la evaluación de los cursos en línea tiene características específicas de acuerdo al modelo andragógico, modular y a distancia. ${ }^{16}$

Los cursos se organizan por objetos de aprendizaje/portafolios en donde se presenta una colección de documentos y actividades, las cuales serán realizadas por los estudiantes para denotar su esfuerzo, progreso y logros. El portafolio es la forma de organizar el proceso de aprendizaje y de evaluación que permite ser monitoreado por el profesor y por el mismo alumno, a través de las aportaciones de producciones de diferente índole por parte del estudiante. ${ }^{17}$

En la evaluación de los trabajos de cada módulo, se toma en cuenta el rescate que el alumno hace de su práctica profesional y expresa en los diferentes momentos de la metodología del trabajo (de la que se habló en el Plan de Estudio antes citado), al construir actividades preliminares, ejercicios y actividades integradoras en las que se pone en juego su capacidad de comprender los nuevos conocimientos, pero y sobre todo, de aplicarlo en situaciones concretas para atender problemas reales de la práctica profesional.

El proceso metodológico de evaluación del programa contempla una cualitativa personalizada que permite retroalimentar el proceso cognitivo del estudiante, y su ponderación cuantitativa que equivale a la calificación que se registra en el kardex.

\footnotetext{
${ }^{14}$ Ver el Reglamento General de Evaluación y Promoción de Alumnos de la Universidad de Guadalajara en http://www.udg.mx/es/normatividad

${ }^{15}$ Ver el Reglamento de la Nivelación de la Licenciatura en Trabajo Social, Sistema Abierto y a Distancia del Programa de Nivelación a la Licenciatura en Trabajo Social en "Carpeta de Apoyo" de cada uno de los doce módulos en Plataforma Moodle

${ }^{16}$ Evidencia en "Tema Cero" de cada uno de los doce módulos en el apartado "Criterios de Evaluación" en Plataforma Moodle.

${ }^{17}$ Evidencia Plataforma Moodle
} 
Para llevar a cabo la evaluación cada una de las academias aplica y determina características y especificaciones propias del dominio teórico, práctico y formativo de los módulos; siendo considerado el instrumento como guía para calificar, pero sin llegar a ser un formato único para expresar las observaciones, sugerencias y resultados a los estudiantes.

Para la toma de decisiones en materia de evaluación de un sistema no presencial y para que ésta no dependa del maestro, sino de criterios estándares que apliquen indistintamente a todos los estudiantes, es indispensable el trabajo colegiado que se realiza a través de las academias. Estas son responsables de construir un instrumento para evaluar los diversos tipos de desempeño (competencias), que alcanzan los estudiantes, diferenciándolos en saberes teóricos, prácticos y formativos, en apego al programa establecido para cada módulo, este instrumento se conoce como rúbrica de evaluación. ${ }^{18}$

La rúbrica de evaluación se utiliza al finalizar el módulo, para evaluar el trabajo entregado por el estudiante, de manera que cualquier maestro de la academia utiliza los mismos aspectos, criterios e indicadores para emitir una calificación final. Estos criterios se ofrecen al estudiante al inicio de cada uno de los módulos.

Un aspecto importante a señalar es que este método de evaluación permite valorar las competencias planteadas en el proyecto curricular, que una vez aprendidas se articulan con las técnicas y conocimientos que los estudiantes operan en su desempeño profesional, que normalmente se relacionan con problemas sociales específicos y que logran evidenciar a través de los productos construidos, bajo las guías de aprendizaje. ${ }^{19}$

Desde luego que la manera en que se evalúa es acorde a los objetivos del plan de estudios, pues éstos no pueden entenderse aislados de los contenidos diseñados para asegurar la adquisición de los diversos conocimientos teórico, prácticos y de formación durante el desarrollo de los aprendizajes autogestivos, de esta manera es posible evaluar los procedimientos académicos, de autogestión, adquisición de competencias, vinculación de conocimientos teórico-prácticos, estrategias, valores, destrezas, actitudes y habilidades deseadas.

No hay que olvidar este programa opera bajo una modalidad no convencional en la que no existe la exposición del docente ni se requiere de la presencia física para la interacción entre docente y alumno. La enseñanza se encauza a través de diferentes medios y la evaluación de los aprendizajes se centra en la capacidad de los alumnos para desenvolverse en esos medios, para resolver y vincular los problemas concretos de la realidad con los contenidos teóricos y en el desarrollo de su autonomía y creatividad.

En cuanto a la evaluación de la construcción social del aprendizaje, o actividades de aprendizaje

\footnotetext{
${ }^{18}$ Evidencia en "Tema Cero" de cada uno de los doce módulos en el apartado "Criterios de Evaluación" en Plataforma Moodle.

${ }^{19}$ Ver Guías de Aprendizaje de los doce módulos en Plataforma Moodle.
} 
colaborativo, podemos afirmar con base en la sistematización de resultados realizados por las academias que operan cada módulo, que el aprendizaje colectivo es mucho más enriquecedor que el individual ya que las actividades realizadas en colaboración ofrecen mayores posibilidades de comprensión, de retroalimentación entre pares, de construcción colaborativa entre compañeros de una misma área de desempeño o de una misma institución en diferentes estados de la República. La inteligencia colectiva puesta en juego y que se ve aumentada por las diferentes edades y años de experiencia de los estudiantes, han posibilitado la realización de interesantes casos integradores cuyos contenidos son operables en diferentes ambientes laborales y en distintas comunidades. ${ }^{20}$

La autoevaluación a que se someten los estudiantes da cuenta de su satisfacción por el aprendizaje logrado entre pares y realizado colaborativamente. Cabe mencionar que la realización de este ejercicio puede ser confrontativo para los estudiantes, no obstante, a la larga manifiestan que esos momentos de reflexión de su propio desempeño, les ayuda a reforzar el compromiso que implica su rol de estudiantes de una licenciatura. ${ }^{21}$

Estos elementos, fortalecen la formación de un perfil de egreso con competencias profesionales integradas, que incluye además de las competencias profesionales/disciplinares, competencias transversales también llamadas para la vida.

Asimismo, la evaluación del proceso formativo apoya y se correlaciona con los objetivos del Proyecto Curricular al facilitar el conocimiento de elementos que generan la reflexión y análisis del quehacer profesional a través de la criticidad de los métodos de trabajo empleados, la aprehensión de referentes teóricos-epistemológicos que expliquen los problemas a los que se enfrenta el estudiante cotidianamente, la búsqueda de nuevos modelos de intervención, así como, fomentar la formación teóricometodológica de los estudiantes del programa, propiciar el desarrollo de la investigación en torno a la práctica profesional del Trabajador Social y capacitar a los estudiantes para analizar y sistematizar su experiencia laboral en cualquier campo de ejercicio profesional en que esté laborando.

En cada módulo el alumno es informado de los procedimientos y criterios de evaluación, definido en las rúbricas y que cada academia establece y aprueba. Los criterios de evaluación serán accesibles al alumno, ya sea en la carpeta denomina "Tema Cero" si trabaja en línea en la Plataforma Moodle: o bien si es modalidad electrónica lo encontrará adjunto a las guías de trabajo y antologías.

La docencia (o proceso de enseñanza) utiliza recursos tecnológicos para la comunicación y el seguimiento del proceso de aprendizaje. Utiliza un diseño instruccional donde el estudiante se ve inmerso

\footnotetext{
${ }^{20}$ Ver Casos Integradores en Plataforma Moodle.

${ }^{21}$ Ver Evaluaciones realizadas por los alumnos al programa, la autoevaluación de los propios alumnos en Plataforma Moodle.
} 
en procesos y ambientes sustentados en dimensiones de aprendizaje, como el perceptual, cognitivo, metodológico, de investigación, colaborativo, meta-aprendizaje, aprendizaje significativo y aprendizaje autogestivo.

El diseño instruccional (guía) es concebido como la ruta crítica que los estudiantes siguen durante el o los procesos de enseñanza-aprendizaje. En él están contenidas las recomendaciones y sugerencias para el trabajo, así como también sirve para regular las interacciones entre los estudiantes, los medios y el asesor. Dicha guía responde a una temática determinada, conocida como objeto de estudio o portafolio de aprendizaje.

Cada objeto de estudio ${ }^{22}$ consta de una serie de elementos que implican, por un lado, indicaciones generales, objetivos, dinámica de trabajo, ejes de problematización, y por otro, aquellas que involucran acciones de los estudiantes: 1) actividad preliminar, recuperación de conocimientos y experiencias previas para propiciar la asimilación de los nuevos aprendizajes. 2) ejercicios, acciones con estrategias para analizar y aplicar información, con el fin de propiciar el diálogo entre los contenidos teóricos y los datos empíricos; y 3) actividad integradora, es la tarea final, estrategias para traducir el análisis en acciones concretas que modifiquen la práctica profesional de nuestros estudiantes. ${ }^{23}$

Al final de los objetos de estudio, la guía presenta las instrucciones para elaborar el caso integrador, en él se trata de vincular y agrupar los aprendizajes parciales de cada uno de los objetos de estudio, para aplicar los conocimientos a la práctica profesional.

Cada módulo tiene un acercamiento general a la metodología y a las técnicas de investigación a través de las actividades que realizan en el mismo. Sin embargo, en los módulos X y XI, relativos a los métodos de investigación social y módulo de integración, se realiza un documento que sustenta las competencias adquiridas sobre estos contenidos de forma específica. ${ }^{24}$

Las modificaciones y/o actualizaciones del plan de estudios o de los programas de las asignaturas de un programa a distancia, implican actualizar continuamente las guías de aprendizaje y las antologías. Este trabajo es realizado por las academias, integradas por profesores titulares adscritos al módulo en cuestión y participan entre 4 y 6 profesores. Una vez realizada, discutida y aprobada la actualización, con su correspondiente bibliografía, es turnada al Colegio Departamental para su aprobación, después se modifican los cursos en línea y posteriormente se socializan con los alumnos. ${ }^{25}$ Cabe mencionar que buena parte de las modificaciones a la forma, sobre todo al diseño instruccional, surgen de la evaluación que hacen los propios alumnos. ${ }^{26}$

\footnotetext{
${ }^{22}$ Guía de Aprendizaje electrónica o en Plataforma Moodle.

${ }^{23}$ Ver las Guías de los doce Módulo que se encuentran en Plataforma Moodle.

${ }^{24}$ Evidencia en las Guías de los módulos X (Plataforma Moodle) y XI (Correo electrónico).

${ }^{25}$ Formato de evaluación del módulo

${ }^{26}$ Evidencias en electrónico en la plataforma MOODLE
} 
De esta forma afirmamos que el programa es efectivo en cuanto a la formación integral de los estudiantes, pues adquieren no sólo competencias que aplican en su área laboral, como son las competencias planeadas en cada uno de los módulos del plan de estudios, las cuales están contenidas en el diseño curricular, sino también competencias para la vida como disciplina, constancia, creatividad, organización del tiempo, mejor manejo de tecnologías, desarrollo de trabajo colaborativo, lo cual se logra gracias a la incorporación de tecnologías de la información y comunicación en los procesos de aprendizaje de nuestros estudiantes.

Lo anterior es evidente al evaluar trabajos finales (casos integradores); en las autoevaluaciones que realizan los estudiantes de su propio proceso en cada módulo, y en las notas que se levantan como registro cualitativo del aprendizaje durante el examen recepcional, ${ }^{27}$ cuando el estudiante se titula por excelencia académica.

Dentro del Departamento de Desarrollo Social se encuentran alojados tres Cuerpos académicos, integrados por los profesores adscritos en este Departamento. Estos cuerpos colegiados han permitido a sus integrantes realizar todo tipo de actividades y vincularlas con cada una de sus academias, de forma que impactan directamente en las actividades sustantivas de docencia, investigación, difusión, con lo cual participan en convocatorias enfocadas a fortalecer el trabajo académico colegiado, la competitividad académica y la calidad de los programas educativos.

\section{A MANERA DE CONCLUSIÓN}

Una evaluación externa permite autoposesionarnos del momento en que nos encontramos desde sus diferentes dimensiones: académica, administrativa, curricular, y tener una visión global acerca del manejo y operación del programa educativo.

Cierto es que ha habido evolución, adaptación a las circunstancias actuales, a las demandas de calidad y pertinencia, a necesidades de asimilación, pero sobre todo a satisfacer las expectativas de los alumnos que eligen estudiar este programa.

Mejoramos nuestros procesos, sistematizamos nuestra información, monitoreamos el paso de los alumnos por el programa y buscamos el egreso eficiente y con calidad, sí, pero estamos en la autoevaluación constante de cómo nos percibimos y como nos perciben nuestros alumnos a fin de que las debilidades no se conviertan en amenazas al programa, sino situaciones de oportunidad en donde podemos crecer, renovarnos con creatividad y en el uso de lo que la tecnología nos ofrece para que lo no convencional adquiera tintes de convencionalidad en una sociedad permanentemente comunicada en lo virtual pero separada en la realidad. En la búsqueda permanente de escuchar las demandas y responder

\footnotetext{
${ }^{27}$ Libretas de secretario de actas de las titulaciones en custodia de la Presidencia del Comité de Titulación.
} 
desde la trinchera institucional y pública pero con calidad reconocida y demostrada. La formación profesional habilitada para el mercado laboral y para la vida, eso queremos, eso ofrecemos. 


\section{References}

2 Tríptico, en físico

3 Dictamen de la nivelación. Evidencia entregada por la coordinación de carrera

4 Reglamento del programa NILITS. Evidencia entregada por la coordinación de carrera

5 Curso propedéutico del programa de nivelación en línea: http://www.cenlinea.cucsh.udg.mx/course/view.php?id=1386

6 Formato de entrevista

7 Proyecto curricular de la nivelación.

8 Proceso de inducción a moodle:

http://www.cenlinea.cucsh.udg.mx/course/view.php?id=1254

9 Ejemplo de acta de valoración del curso propedéutico.

10 Reglamento de la Nivelación en Trabajo Social

11 Dictámenes y cuadro de porcentaje de aspirantes por ciclo escolar.

12Programa de tutoría de NILITS, evidencia en la coordinación de carrera

13Cursos en plataforma y actas de academia, evidencia en la coordinación de docencia.

14 Ver el Reglamento General de Evaluación y Promoción de Alumnos de la Universidad de Guadalajara en http://www.udg.mx/es/normatividad

15 Ver el Reglamento de la Nivelación de la Licenciatura en Trabajo Social, Sistema Abierto y a Distancia del Programa de Nivelación a la Licenciatura en Trabajo Social en "Carpeta de Apoyo" de cada uno de los doce módulos en Plataforma Moodle.

16 Evidencia en "Tema Cero" de cada uno de los doce módulos en el apartado "Criterios de Evaluación" en Plataforma Moodle.

17 Evidencia Plataforma Moodle.

18 Evidencia en "Tema Cero" de cada uno de los doce módulos en el apartado "Criterios de Evaluación" en Plataforma Moodle.

19 Ver Guías de Aprendizaje de los doce módulos en Plataforma Moodle.

20 Ver Casos Integradores en Plataforma Moodle.

21 Ver Evaluaciones realizadas por los alumnos al programa, la autoevaluación de los propios alumnos en Plataforma Moodle. 
22 Guía de Aprendizaje electrónica o en Plataforma Moodle.

23 Ver las Guías de los doce Módulo que se encuentran en Plataforma Moodle.

24 Evidencia en las Guías de los módulos X (Plataforma Moodle) y XI (Correo electrónico).

25 Formato de evaluación del módulo

\section{Evidencias en electrónico en la plataforma MOODLE}

27 Libretas de secretario de actas de las titulaciones en custodia de la Presidencia del Comité de Titulación. 